Systematic Distortions in Musicians' Reproduction of Cyclic Three-Interval Rhythms Author(s): Repp Bruno H., London Justin, Peter E. Keller

Source: Music Perception: An Interdisciplinary Journal, Vol. 30, No. 3 (February 2013), pp. 291-305

Published by: University of California Press

Stable URL: http://www.jstor.org/stable/10.1525/mp.2012.30.3.291

Accessed: 22/01/2015 03:49

Your use of the JSTOR archive indicates your acceptance of the Terms \& Conditions of Use, available at http://www.jstor.org/page/info/about/policies/terms.jsp

JSTOR is a not-for-profit service that helps scholars, researchers, and students discover, use, and build upon a wide range of content in a trusted digital archive. We use information technology and tools to increase productivity and facilitate new forms of scholarship. For more information about JSTOR, please contact support@ jstor.org. 


\section{Systematic Distortions in Musicians' Reproduction of Cyclic Three-Interval Rhythms}

BRUNO H. REPP

Haskins Laboratories, New Haven, Connecticut

JUSTIN LONDON

Carleton College

Peter E. Keller

Max Planck Institute for Human Cognitive and Brain Sciences, Leipzig, Germany

IN A CLASSIC STUDY, FrAISSE (1956) DEMONSTRATED that sequences of four sounds defining three different interval durations exhibit characteristic distortions in reproduction: The two more similar intervals tend to be assimilated to each other, resulting in a rhythm containing just two interval durations. The present study examined whether highly trained musicians (including percussionists) are able to perform such rhythms accurately in a synchronization-continuation tapping paradigm. Eleven rhythms, a subset of those used by Fraisse, were presented cyclically at his original tempo and also at a slower tempo. The musicians produced significant rhythm distortions, though they were smaller than those observed by Fraisse and not always assimilative. They were relatively larger at the fast than at the slow tempo and occurred in both synchronization and continuation. In contrast to Fraisse's data, the most variably reproduced target rhythm was the one in which the two longer intervals were identical. The pattern of distortions suggested attraction towards ideal rhythms in which all three interval durations are different, representing metrical categories with nominally simple interval ratios (some permutation of $1: 2: 3$ ) that were probably activated by the cyclic presentation of the rhythms. However, these attractors themselves seemed to be somewhat distorted, perhaps reflecting the simultaneous presence of a nonmetrical attractor that differentiated two interval categories regardless of ratio, as observed by Fraisse.

Received: November 9, 2011, accepted May 27, 2012.

Key words: rhythm production, interval ratios, synchronization, assimilation, attractor
$\mathrm{W}$ ESTERN MUSICAL RHYTHMS USUALLY FIT a simple metrical scheme according to which the intervals between regular beats are subdivided into two or three equal parts that in turn may be subdivided in a similar manner (London, 2004). The temporal positions in the resulting metrical hierarchy may or may not be occupied by sound onsets, and in this way a composer or experimenter can generate rhythms varying in complexity. When rhythms are actually performed by a musician, however, they often deviate considerably from the simple interval ratios that subdivision by two or three implies (e.g., Gabrielsson, Bengtsson, \& Gabrielsson, 1983). These "expressive" deviations give the rhythm a certain character, as may be required by a particular musical style. Expressive rhythms, in which the interval durations may have quite complex ratios, do not seem difficult to produce, at least not by expert musicians. Nevertheless, controlled laboratory experiments with rhythms harboring various interval ratios reveal that some rhythms are much easier to reproduce accurately than others and that simple interval ratios do play an important role in reproduction.

When a rhythm composed of two different interval durations $(\mathrm{i} 1<\mathrm{i} 2)$ is presented cyclically to participants who are asked to tap along with it and/or to reproduce it exactly, characteristic distortions are observed that depend on the $\mathrm{i} 1: \mathrm{i} 2$ ratio (or $\mathrm{i} 1 / \mathrm{i} 2$ fraction). When the ratio is smaller than $1: 2(=0.5)$, participants typically increase it by making the two intervals more similar to each other (assimilation). When the ratio is larger than $1: 2$, participants tend to decrease it in reproduction by increasing the contrast between the two intervals (Povel, 1981; Summers, Bell, \& Burns, 1989; Summers, Hawkins, \& Mayers, 1986). These distortions suggest an optimal interval ratio or "attractor" in the vicinity of $1: 2$, in accord with an underlying triple meter $(1+1$ $+1)$. The optimal ratio is defined as the ratio that would be reproduced without any distortion, on average, and presumably also with the lowest variability. It is an attractor in the sense that other rhythms diverge in its direction, though usually without reaching it completely. Even highly trained musicians tapping in synchrony with an exact auditory rhythm template show these

Music Perception, volume 30, issue 3, pp. 291-305, issn 0730-7829, electronic issn 1533-8312. C 2013 by the Regents of the university of California all RIGHTS RESERVED. PLEASE DIRECT ALL REQUESTS FOR PERMISSION TO PHOTOCOPY OR REPRODUCE ARTICLE CONTENT THROUGH THE UNIVERSITY OF CALIFORNIA PRESS'S RIGHTS AND PERMISSIONS WEBSITE, HTTP://WWW.UCPRESSJOURNALS.COM/REPRINTINFO.ASP. DOI: 10.1525/MP.2012.30.3.291 
distortions, which thus seem to be almost unavoidable. Recent investigations have suggested that the attractor for musicians is not exactly 1:2 but slightly larger, whereas it is often smaller than 1:2 for nonmusicians (Repp, London, \& Keller, 2011, 2012). The reasons for these deviations from the exact simple (i.e., small-integer) ratio are not yet fully understood. However, there is evidence from studies of rhythm categorization that the center (presumably the most representative instance) of a rhythm category is often not located exactly at the simplest interval ratio (Desain \& Honing, 2003; Sadakata, Desain, \& Honing, 2006). Nevertheless, rhythm categories are generally described and notated in terms of simple interval ratios.

The purpose of the present study was to investigate in a preliminary way whether there are any simple attractors (i.e., having simple interval ratios) in cyclic reproduction of rhythms composed of three intervals (i1, i2, i3), and what they might be. The presence of attractors would be indicated by systematic distortions in rhythm reproduction that move the produced rhythm closer (but not necessarily all the way) to the hypothetical attractor in the "rhythm space" defined by the three interval durations. Certainly, the isochronous 1:1:1 category must be one attractor, but it probably plays only a minor role when the target rhythm is perceptibly non-isochronous, so participants know they have to produce a non-isochronous rhythm. Other candidates for simple attractors are 1:1:2 and its permutations, which fit a duple $(2+2)$ meter (Desain \& Honing, 2003). Much less likely candidates are 1:2:2 and its permutations, which suggest a quintuple or uneven $(3+2$ or $2+3$ ) meter, which is uncommon in Western music. Indeed, participants in Desain and Honing's study did not use these categories at all when transcribing various auditory rhythms having complex interval ratios, whereas they did use categories composed of three different interval durations (permutations of 1:2:3), which are compatible with either a triple $(2+2+2)$ or compound duple $(3+3)$ meter. Given these categorization results, one might predict that, when trying to reproduce a rhythm composed of three different intervals having complex ratios with each other, participants will either distinguish all three intervals (perhaps with distortions suggesting attraction to a 1:2:3 relationship) or will tend to assimilate the two shorter intervals to each other (i.e., be attracted to 1:1:2), but not the two longer intervals (which would imply attraction to $1: 2: 2)$. This prediction is not borne out by existing data, however.

As with so many other aspects of rhythm and timing, pioneering research on three-interval rhythms was

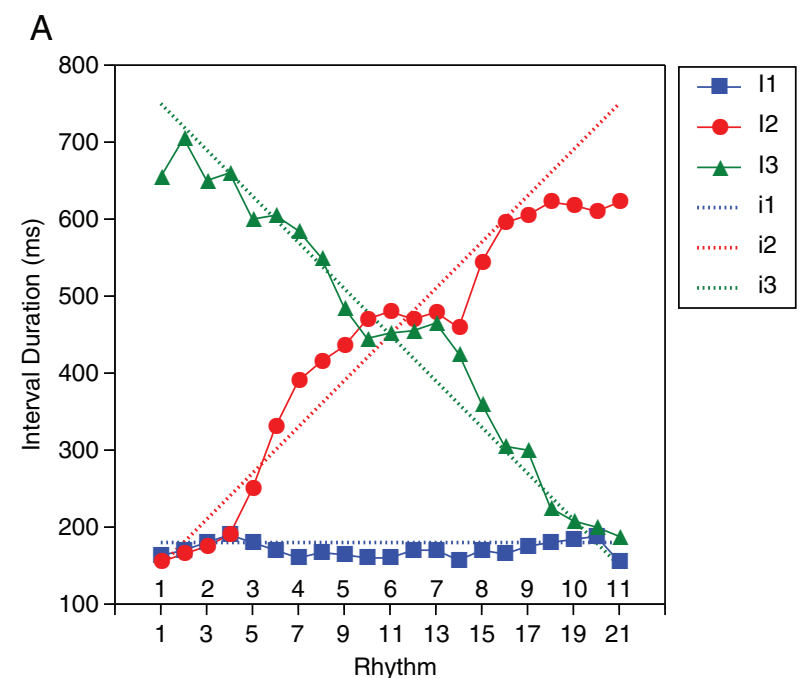

B

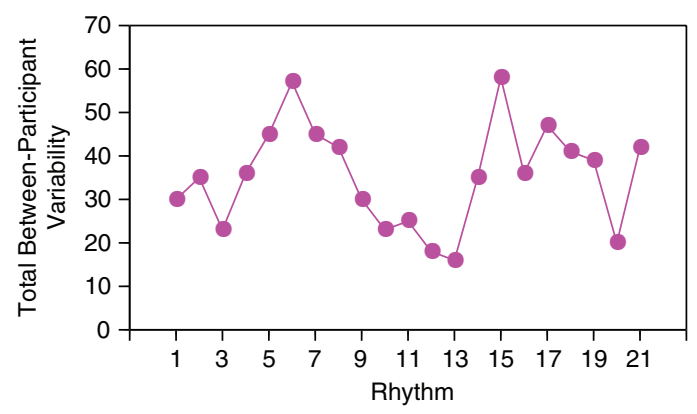

FIGURE. 1 Results of Fraisse's (1956) Experiment 3, copied and redrawn from his Figure 10 (p. 55). Panel A shows mean produced interval durations $(11,12,13)$ in response to target rhythms with intervals $i 1, i 2$, i3 (dotted lines). Panel B shows a measure of interindividual variability for each rhythm (all three intervals combined). Each rhythm was reproduced once by each of 10 participants. The numbers at the bottom in panel $A$ indicate the rhythms we selected for our experiment.

carried out by Fraisse (1956). ${ }^{1}$ Importantly, Fraisse did not present rhythms cyclically but as isolated rhythmic groups. Thus, a three-interval rhythm was articulated by a sequence of four sounds. This pattern was presented once, or several times with pauses between presentations, before participants reproduced it (again, once or several times, in the latter case pausing between groups). In the experiment that served as the model for the present study (his Experiment 3), Fraisse created a set of 21 rhythms by holding il constant at $180 \mathrm{~ms}$ and varying i2 and $\mathrm{i} 3$ in a complementary fashion between 150 and $750 \mathrm{~ms}$ in steps of $30 \mathrm{~ms}$, so that their sum was always $900 \mathrm{~ms}$, as shown by the dotted lines in Figure 1A. The

\footnotetext{
${ }^{1}$ An English translation of the relevant chapter from that monograph (Chapter 4) can be obtained from author BHR. All subsequent references to Fraisse without date are to that chapter.
} 
mean produced interval durations (denoted here by I1, I2, I3) are given as symbols connected by solid lines. Within the central range of this rhythm continuum, where i2 and i3 were roughly between 300 and 600 $\mathrm{ms}, \mathrm{I} 2$ and I3 tended to be assimilated to each other, while I1 fell slightly short of i1. Thus, it was the two longer intervals that were assimilated, not the two shorter intervals. Only near the ends of the rhythm continuum, where either i2 or i3 became similar to i1 in duration, assimilation of these two short intervals occurred instead, together with some shortening of the long interval. Interindividual variability, shown in Figure $1 \mathrm{~B}$, was maximal near the boundary between these two assimilation regions. The principle that Fraisse derived from these results is that there is a strong preference for having only two distinct interval durations in a rhythm; therefore, the two more similar intervals in a three-interval rhythm tend to be assimilated to each other. Fraisse did not consider the possible role of meter or of attractors with simple interval ratios, and indeed they may not have played a role in his experiment because the rhythms were not presented cyclically.

Some more recent studies have examined cyclic (re)production of three-interval rhythms. Repp, Windsor, and Desain (2002) presented pianists with notated metrical rhythms in which the three interval durations were always related in some permutation of 1:2:3. Pianists had to play melodies instantiating these rhythms repeatedly, at four different tempi. Even though the target ratios were simple, the two longer intervals tended to be assimilated to each other in production, whereas the short interval was produced fairly accurately (i.e., as $1 / 6$ of the cycle duration). This pattern of results seems consistent with Fraisse's findings, where the two longer intervals were assimilated to each other as long as their ratio was larger than $1: 2$. (Note that $2: 3=0.67$ is larger than $1: 2=0.5$.) However, the assimilation observed by Repp et al. was only a tendency that rarely resulted in complete assimilation; moreover, it depended on interval order and on the notated meter, and it increased with tempo.

Repp, London, and Keller (2005) used a cyclic rhythm reproduction task. Musically trained participants first tapped in synchrony with a cyclic auditory template of the rhythm and then continued to tap the rhythm in self-paced and metronome-paced conditions, at a range of rather fast tempi. The three target intervals in that study had ratios of 2:2:3 or 2:3:3, in all possible permutations; thus they had only two distinct durations. The reproductions revealed a general tendency to increase the contrast between the two interval durations, especially in the 2:2:3 rhythms. This tendency was as large in synchronization as in continuation tapping, and it increased with tempo. Subsequent studies still found similar distortions at slower tempi (Repp, London, \& Keller, 2008; Snyder, Hannon, Large, \& Christiansen, 2006). While these results also seem consistent with Fraisse's principle that threeinterval rhythms gravitate towards two distinct interval categories whose durations do not necessarily form a simple ratio, for the $2: 2: 3$ rhythms the tendency to enlarge the difference between interval durations could also reflect attraction towards a 1:1:2 relationship among the intervals, and perhaps towards the simpler meter that goes with it. No recent study has focused on reproduction of three-interval rhythms having arbitrarily complex interval ratios, as in Fraisse's pioneering work.

The aim of the present study was to investigate whether the interval assimilation tendencies observed in Fraisse's experiment with single-cycle rhythms having three different interval durations with arbitrary ratios (Figure 1A) are sufficiently general to be observed also in a cyclic rhythm reproduction task, and sufficiently coercive to be exhibited even by highly trained musicians, including percussionists. In other words, can arbitrary three-interval rhythms be reproduced accurately in a cyclic paradigm? Cyclic rhythm production is very common in music performance, where the same rhythm is often maintained for many measures, especially in dance or dance-derived music. By contrast, Fraisse's task of reproducing a single rhythmic group following an auditory model is less typical of musical pursuits. Musicians, unlike Fraisse's participants, are highly skilled in rhythm production, with percussionists being true experts. ${ }^{2}$ We asked them to synchronize finger taps with an exact auditory rhythm template and then to continue to tap the rhythm on their own. If we succeeded in demonstrating that systematic (though perhaps small) distortions in three-interval rhythm reproduction still occur under those stringent conditions, this would demonstrate the almost obligatory nature of the distortions and might indicate the operation of attractors in rhythm space. We considered it possible that we would find a somewhat different pattern of distortions than Fraisse did because in cyclic rhythms metrical structure may play a role. However, we did not attempt to manipulate meter and left any possible metrical interpretation of the rhythms to the participants.

\footnotetext{
${ }^{2}$ Since Fraisse does not mention music training, we presume his participants were not musicians.
} 
We used a subset of Fraisse's rhythms and presented it both at his original tempo and at a slower tempo. In discussing rhythmic structures, Fraisse (1956, Chapter 2) distinguished between categories of short and long intervals, with the boundary being around $300-400 \mathrm{~ms}$, and claimed that assimilation occurs within these categories in production, whereas contrast occurs between them. We wondered whether the same pattern of distortions would still be observed if none of the three intervals in the rhythm was really short in Fraisse's scheme. Such a result would suggest either that the assimilation tendencies do not derive from a contrast between categories of short and long intervals or that the short-long category boundary is relative and shifts with tempo. Other results, reviewed earlier, made us expect that rhythm distortions would generally be relatively smaller at a slow than at a fast tempo.

\section{Method}

\section{PARTICIPANTS}

The 14 participants were divided into two groups that we will call musicians and rhythm experts. The musicians $(N=9)$ included 8 graduate students from the Yale School of Music (2 men, 6 women, ages 21-27) and author BHR (age 66). The young professional musicians had studied their primary instruments (piano-2, viola-2, flute, trombone, harp, guitar) for 10-24 years. BHR is a lifelong amateur pianist with 10 years of instruction in childhood and has much experience with tapping experiments; his tapping performance is usually similar to that of young musicians. The rhythm experts $(N=5$, all men, ages 23-27) included three graduate students of percussion from the Yale School of Music, one doctoral student of percussion from the Hartt School of Music, and one graduate student of violin from Yale whose exceptional rhythmic acuity was known from previous experiments and who was therefore grouped with the percussionists. Years of instruction of the rhythm experts ranged from 12 to 20 . All young participants were paid for their participation. All participants were right-handed by self-report.

\section{EQUIPMENT AND MATERIALS}

The experiment was controlled by a program written in Max/MSP 4.0.9 and running on an Intel iMac computer. All tones were produced by a Roland RD-250s digital piano, had a nominal (MIDI) duration of $40 \mathrm{~ms}$, and the same intensity (MIDI velocity). Participants listened over Sennheiser HD280 pro headphones at a comfortable loudness and tapped on a Roland SPD6 percussion pad that they held on their lap.
TABLE 1 Interval Durations (ms) and Their Ratios in the Rhythms Used.

\begin{tabular}{lccccccl}
\hline & $\begin{array}{c}\mathrm{i} 1 \\
\text { (fast) }\end{array}$ & $\begin{array}{c}\mathrm{i} 2 \\
\text { (fast) }\end{array}$ & $\begin{array}{c}\mathrm{i} 3 \\
\text { (fast) }\end{array}$ & $\begin{array}{c}\mathrm{i} 1 \\
\text { (slow) }\end{array}$ & $\begin{array}{c}\mathrm{i} 2 \\
\text { (slow) }\end{array}$ & $\begin{array}{c}\mathrm{i} 3 \\
\text { (slow) }\end{array}$ & $\mathrm{i} 1: \mathrm{i} 2: \mathrm{i} 3$ \\
\hline $\mathrm{R} 1$ & 180 & 150 & 750 & 450 & 375 & 1825 & $6: 5: 25$ \\
$\mathrm{R} 2$ & 180 & 210 & 690 & 450 & 525 & 1675 & $6: 7: 23$ \\
$\mathrm{R} 3$ & 180 & 270 & 630 & 450 & 675 & 1525 & $2: 3: 7$ \\
$\mathrm{R} 4$ & 180 & 330 & 570 & 450 & 825 & 1375 & $6: 11: 19$ \\
R5 & 180 & 390 & 510 & 450 & 975 & 1225 & $6: 13: 17$ \\
R6 & 180 & 450 & 450 & 450 & 1075 & 1075 & $2: 5: 5$ \\
R7 & 180 & 510 & 390 & 450 & 1225 & 975 & $6: 17: 13$ \\
R8 & 180 & 570 & 330 & 450 & 1375 & 825 & $6: 19: 11$ \\
R9 & 180 & 630 & 270 & 450 & 1525 & 675 & $2: 7: 3$ \\
R10 & 180 & 690 & 210 & 450 & 1675 & 525 & $6: 23: 7$ \\
R11 & 180 & 750 & 150 & 450 & 1825 & 375 & $6: 25: 5$ \\
\hline
\end{tabular}

Each rhythm was played as a cyclically repeated sequence of three tones with pitches C4, D4, and E4. The constant short interval, il, occurred between the onsets of the C4 and D4 tones and was $180 \mathrm{~ms}$ long at the fast tempo (the one also used by Fraisse) and 450 $\mathrm{ms}$ long at the slow tempo. The i2 and i3 intervals occurred between D4 and E4 and between E4 and C4, respectively. At the fast tempo, their durations ranged from $150 / 750 \mathrm{~ms}$ to $750 / 150 \mathrm{~ms}$ in steps of $60 \mathrm{~ms}$, which resulted in 11 different rhythms. (Fraisse had used the same range with steps of $30 \mathrm{~ms}$; the rhythms we selected are numbered at the bottom of Figure 1A.) At the slow tempo, their durations ranged from 375/1875 ms to $1875 / 375 \mathrm{~ms}$ in steps of $150 \mathrm{~ms}$. Cycle durations for all rhythms were $1080 \mathrm{~ms}$ (fast) and $2700 \mathrm{~ms}$ (slow). The rhythms at the fast and slow tempi represented the same (more or less complex) interval ratios, as they merely differed by a scale factor of 2.5 . Table 1 lists the interval durations and ratios of all rhythms, while Figure 2 shows schematic time-line representations of Rhythms 1,6 , and 11 in the fast set. The three tones separated by the two shorter intervals were likely to form a rhythmic group in perception, with the long interval separating successive groups, as indicated in the figure. Near the center of the rhythm continuum, however, rhythmic grouping was more ambiguous.

At the fast tempo, each trial consisted of 23 auditory rhythm cycles followed by a silent interval for continuation tapping equal in duration to 20 cycles. At the slow tempo, where trials were kept to approximately the same length, 9 auditory rhythm cycles were followed by 8 silent cycles. The continuation interval was terminated by a single tone, the signal to stop tapping. Each trial block consisted of 11 randomly ordered trials, representing the 11 different rhythms at the same tempo. 


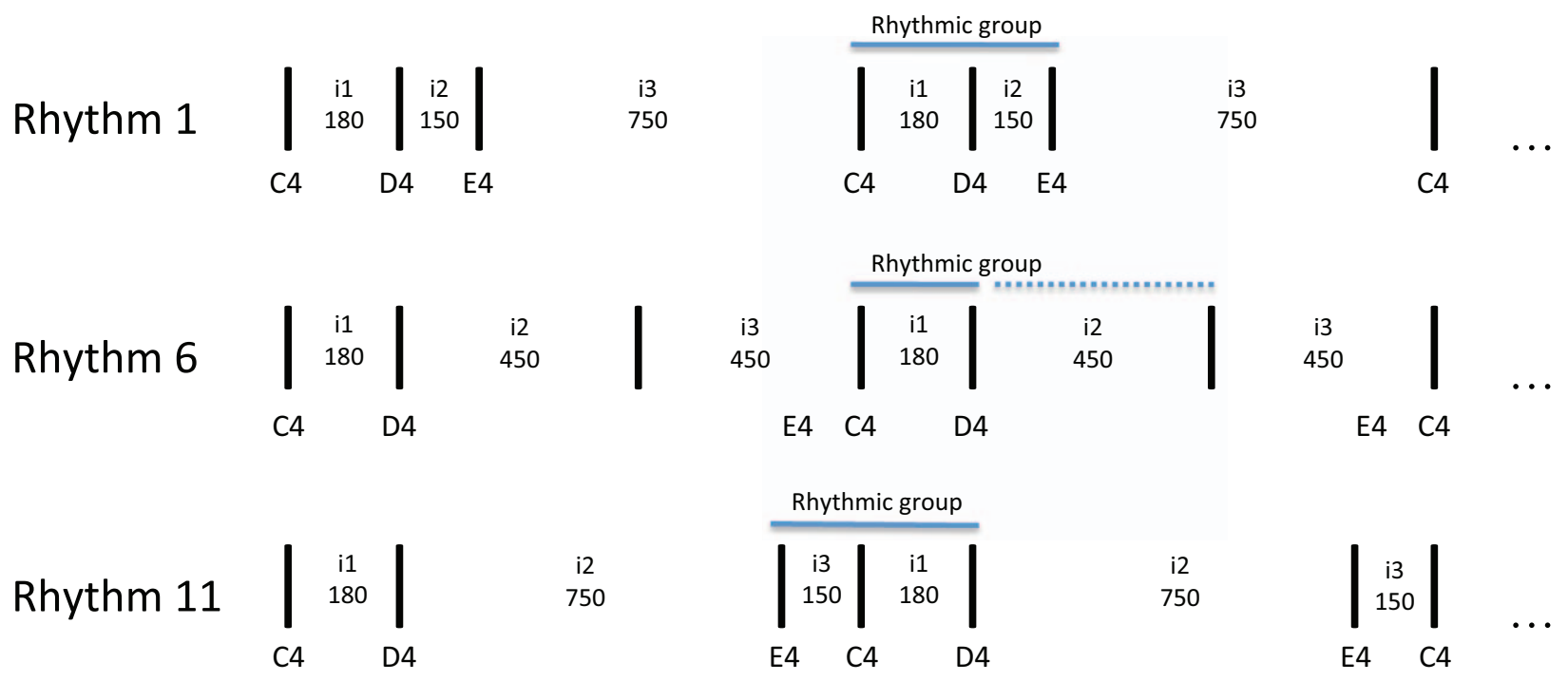

FIGURE. 2 Schematic diagram of Rhythms 1, 6, and 11 from the set we used (two cycles of each). Vertical bars represent tones, i1, i2, i3 represent intervals, small numbers represent interonset interval (IOI) durations in milliseconds at the fast tempo, and C4, D4, E4 represent pitches. Likely rhythmic grouping is indicated; the dotted line suggests uncertainty.

\section{PROCEDURE}

The two tempo conditions were run in separate 1-hour sessions that were separated by at least one day, more often by one week or more. Their order was counterbalanced. Each session consisted of five trial blocks. Participants sat in front of the computer and started each trial by pressing the space bar. The rhythm started $2 \mathrm{~s}$ later. Participants were instructed to start tapping with the third rhythm cycle and to tap with the left hand on C4 and with the right hand on D4 and E4, using the upper left and upper right segments of the percussion pad. $^{3}$ When the auditory sequence stopped, they were to continue tapping the rhythm without interruption until they heard the signal to stop tapping. The style of tapping was not prescribed; most participants moved their arms rather than a single finger, but contact with the pad had to be made with a single finger. The finger impacts were audible as thuds, especially during continuation tapping. The importance of accuracy of synchronization

\footnotetext{
${ }^{3}$ Tapping was divided between hands to avoid having to make three taps in rapid succession with the same hand, which might have been difficult for some participants. (Participants in Fraisse's experiment had tapped unimanually.) There is evidence that, in the absence of biomechanical difficulties, unimanual and bimanual tapping of rhythms is timed very similarly (Semjen \& Ivry, 2001; Summers, Bell, \& Burns, 1989). Occasionally participants started tapping too soon or too late. This was no problem, as we analyzed only taps starting with the fifth cycle. When i3 was short, participants often started tapping with the E4 tone, which suggests that they grouped it with the following tones (cf. Figure 2).
}

and of continuing the exact rhythm at the same tempo was stressed.

\section{ANALYSIS}

The analysis focused on intertap interval durations (I1, I2, I3) and variability. ${ }^{4}$ We first computed the means and within-trial standard deviations ( $S D-\mathrm{w}$ values) of these intervals across cycles in each trial, separately for the synchronization and continuation tasks. The first two synchronization cycles (i.e., the taps synchronized with the third and fourth rhythm cycles) and late continuation cycles were omitted. At the fast tempo, the means and $S D$-w values for synchronization and continuation were each based on 19 cycles (sometimes fewer for continuation if a participants slowed down during continuation). At the slow tempo, they were based on 5 cycles for synchronization and 7 cycles for continuation. Subsequently, the means and betweentrial $S D$ s ( $S D$-b values) of the mean interval durations were calculated across the five trials representing the same rhythm (i.e., across trial blocks). Whereas the $S D-\mathrm{w}$ is a measure of rhythm stability from cycle to cycle, the $S D$-b is a measure of variability in participants' rhythm "interpretation" (i.e., how they time the rhythm on average) from trial to trial.

\footnotetext{
${ }^{4}$ A small amount of data was lost due to skipped trials $(0.5 \%$ of all trials) and missing taps within trials ( $0.2 \%$ of all taps). Rare extra taps were deleted. Occasionally a participant strayed off the prescribed tapping pads, but those taps were counted as correct.
} 
For display in graphs only, deviations of the mean produced interval durations of each rhythm from the target values were assessed by two-tailed $t$-tests separately for each participant group, without any correction for multiple comparisons. For more thorough statistical analysis, each participant's mean interval durations (I1, I2, I3) for the 11 rhythms were expressed as signed deviations from the target values (i2, i2, i3). To make deviations at the fast and slow tempi more comparable, the latter were divided by 2.5 , as the target durations were 2.5 times larger at the slow than at the fast tempo. Similarly, the $S D$-b and $S D-$ w values for the slow tempo were divided by 2.5 for the statistical analyses. Thus all values were normalized relative to target cycle duration (tempo). These relative interval deviations, $S D-\mathrm{b}$, and $S D-\mathrm{w}$ values for each of the three intervals (I1, I2, I3) were then submitted to nine separate $2 \times(2 \times 2 \times 11)$ mixed-model ANOVAs. The between-participant variable was expertise (musicians, rhythm experts), and the within-participant variables were tempo (fast, slow), task (synchronization, continuation), and rhythm (11 levels). To clarify interactions involving tempo, if necessary, each ANOVA was followed by two $2 \times(2 \times 11)$ ANOVAs, one for each tempo. The Greenhouse-Geisser correction was applied to the $p$-level of all effects involving rhythm. These effects were also decomposed into single degree of freedom polynomial contrasts (linear, quadratic, cubic, and higher order up to $10^{\text {th }}$ ).

To quantify the overall deviation of the produced rhythms from the rhythm templates, we further conducted a Procrustes analysis (Dryden \& Mardia, 1998) that calculated the minimal distance between the mean produced intervals and the target intervals, for all rhythms and intervals combined. This distance was determined for each participant in each Tempo $\times$ Task condition, and these data were then submitted to a mixed-model $2 \times(2 \times 2)$ ANOVA with the between-participant variable of expertise and the within-participant variables of tempo and task.

\section{Results}

\section{MEAN INTERVAL DURATIONS}

Figure 3 presents the mean interval durations at the fast tempo in the same format as Figure 1A, so the results can be compared directly with Fraisse's data. Figure 4 shows the corresponding data at the slow tempo. Results for synchronization and continuation were quite similar at each tempo. While deviations from the target intervals were much smaller here than in Fraisse's study, both participant groups showed a number of significant ( $p<.05$, two-tailed) deviations from the target durations, which are indicated by color-coded letters in the figure ( $\mathrm{S}=$ synchronization; $\mathrm{C}=$ continuation). These deviations tended to be smaller for the rhythm experts than for the musicians, but the pattern of deviations was similar for the two groups. Deviations were also relatively smaller and less often significant at the slow than at the fast tempo.

In the ANOVAs on the relative deviations of the interval durations from their target values, the main effect of rhythm and its interactions with other variables were of primary interest. A significant main effect of rhythm would indicate that deviations varied systematically across the 11 rhythms and were not just due to random variability. If only the linear contrast were significant, however, this would indicate merely some dependence of deviations on interval duration, at least for I2 and I3. Therefore, the real indicators of interesting rhythm effects were the higher-order nonlinear contrasts. We now describe the results separately for each interval.

For I1, even though it had a constant short target duration, the main effect of rhythm was significant, $F(10,120)=7.86, p<.001$. Its linear contrast was not significant, but six of the nine nonlinear contrasts were significant $(p<.05)$, the most consistent effect being of $7^{\text {th }}$ order, $F(1,12)=64.59, p<.001$. This indicates a complex and highly reliable pattern of deviations. Rhythm also interacted with expertise, $F(10,120)=2.87$, $p=.03$, with tempo, $F(10,120)=4.16, p=.002$, with task, $F(10,120)=7.63, p<.001$, and with tempo and task, $F(10,120)=3.64, p=.008$. The first three effects can be interpreted, respectively, as reflecting larger relative deviations in musicians than in rhythm experts, larger relative deviations at the fast tempo than at the slow tempo, and larger relative deviations in synchronization than in continuation (see Figures 3 and 4). The triple interaction seemed to be due to a less pronounced Task $\times$ Rhythm interaction at the fast tempo, $F(10,120)=3.73$, $p=.004$, than at the slow tempo, $F(10,120)=$ $7.02, p<.001$. The main effect of rhythm was still significant at the slow tempo, $F(10,120)=4.53, p=$ .002 , with the $7^{\text {th }}$ order contrast still being most pronounced, $F(1,12)=43.33, p<.001$. Of the effects not involving rhythm in the overall ANOVA, only the main effect of tempo reached significance, $F(1$, $10)=8.54, p=.01$. It reflects a tendency for I1 to fall short of i1 at the fast tempo, whereas at the slow tempo the deviations tended to go in the opposite direction. 
A

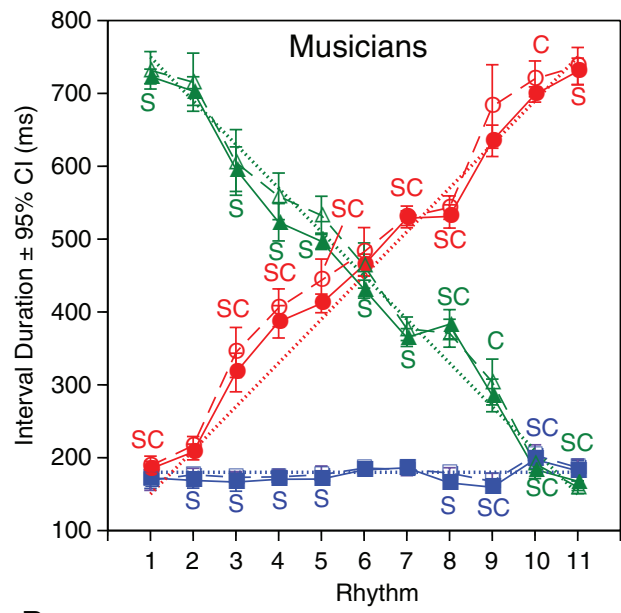

B

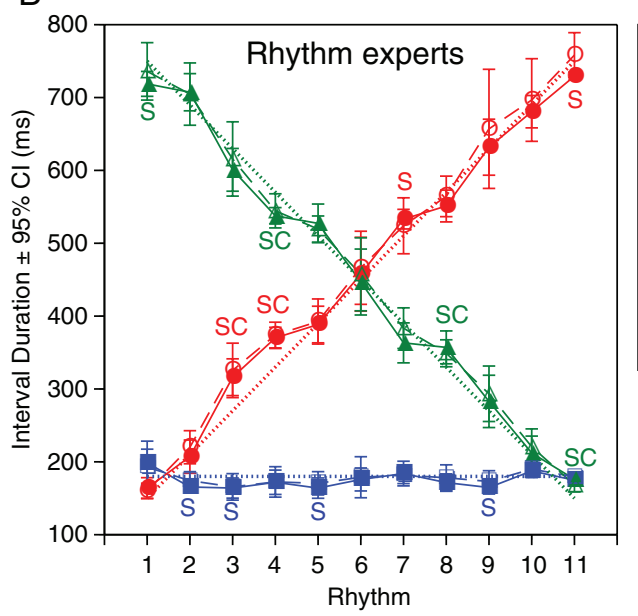

FIGURE. 3 Mean interval durations produced by musicians (panel A) and rhythm experts (panel B) in synchronization (S) and continuation (C) at the fast tempo. Error bars represent $95 \%$ confidence intervals, and capital letters indicate significant $(p<.05)$ differences of the corresponding produced intervals from their target durations.

For I2, the main effect of rhythm was highly reliable, $F(10,120)=10.40, p<.001$. Eight of the ten polynomial contrasts (including the linear one) reached significance, with the most reliable contrasts being $9^{\text {th }}$ order, $F(1,12)=43.49, p<.001$, and $7^{\text {th }}$ order, $F(1,12)=$ $25.06, p<.001$. The linear trend reflects a tendency to overshoot i2 as its duration increased. Rhythm interacted only with tempo, $F(10,120)=7.63, p<.001$, due to larger relative deviations at the fast tempo. The main effect of rhythm was still significant at the slow tempo, $F(10,120)=3.95, p=.004$, and exhibited reliable $7^{\text {th }}$ and $9^{\text {th }}$ order contrasts, $F(1,12)=33.17, p<.001$, and 11.97 , $p=.005$, respectively. Of the effects not involving rhythm, only the main effect of task was marginally
A

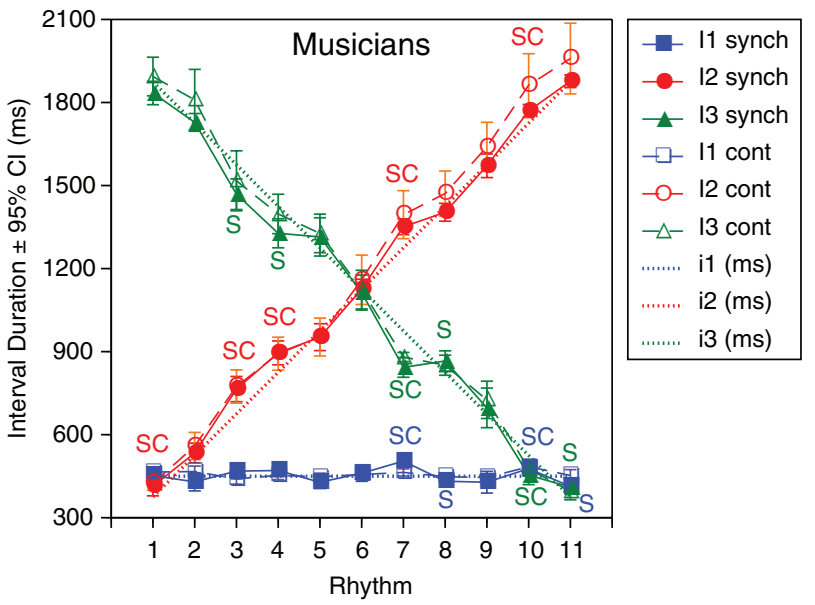

B

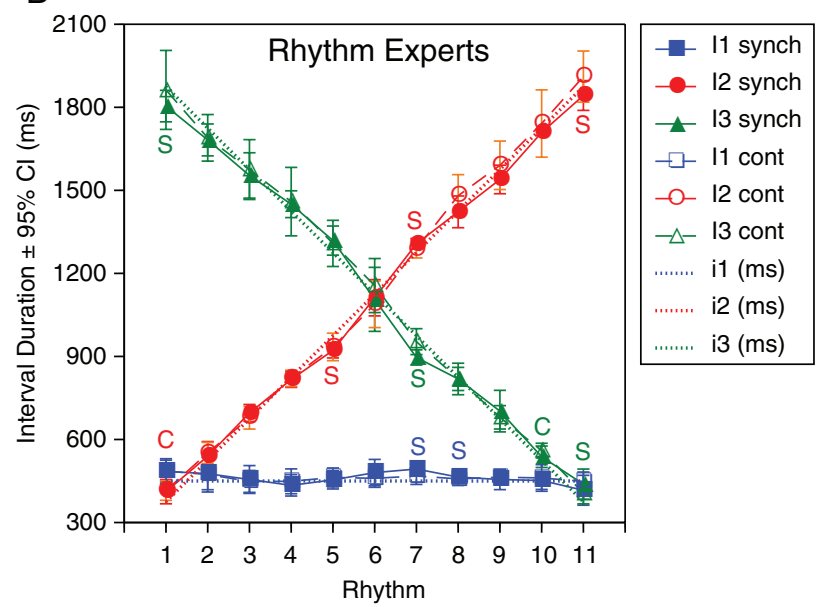

FIGURE. 4 Mean interval durations produced by musicians (panel A) and rhythm experts (panel B) in synchronization (S) and continuation (C) at the slow tempo. Error bars represent $95 \%$ confidence intervals, and capital letters indicate significant $(p<.05)$ differences of the corresponding produced intervals from their target durations.

significant, $F(1,12)=5.12, p=.04$, due to larger positive deviations in continuation than in synchronization. ${ }^{5}$

For I3, where the effects of rhythm were largely complementary to those for I2, the main effect of rhythm was again significant, $F(10,120)=11.84, p<.001$, due mainly

\footnotetext{
${ }^{5}$ This may reflect a slight slowing of tempo during continuation. However, it is not clear whether tempo (cycle duration) was controlled directly by the participants or merely emerged as the sum of produced interval durations. Instead of comparing raw interval durations with the target values (as Fraisse did), we could have compared tempo-normalized interval durations to the interval targets, or interval ratios to ratio targets. The conclusions would have been similar, however, as tempo deviations during continuation tapping were relatively small. During synchronization the tempo was accurate, of course.
} 
to large $7^{\text {th }}$ and $9^{\text {th }}$ order contrasts, $F(1,12)=56.26$ and 41.10 , respectively, both $p<.001$. Rhythm interacted weakly with expertise, $F(10,120)=2.57, p=$ .04 , due to smaller deviations in rhythm experts than in musicians. Rhythm also interacted with tempo, $F(10,120)=2.75, p=.02$, reflecting larger relative deviations at the fast than at the slow tempo, and with task, $F(10,120)=4.42, p=.003$, an interaction located mainly in the $9^{\text {th }}$ order contrast, $F(1,12)=25.72, p<$ .001 , which was more pronounced at the fast tempo. The main effect of rhythm was still significant at the slow tempo, $F(10,120)=4.93, p=.001$, mainly due to a strong $7^{\text {th }}$ order contrast, $F(1,12)=36.06, p<.001$. Of the effects not involving rhythm, again only the main effect of task was marginally significant, $F(1,12)=$ $5.18, p=.04$, reflecting more positive deviations during continuation than during synchronization.

Let us now compare the pattern of deviations at the fast tempo (Figure 3) with the data obtained by Fraisse (Figure 1A). We will refer to individual rhythms as R1-R11 (see Table 1 and numbers at the bottoms of figures). Overall, deviations were much smaller here than in Fraisse's study, which is likely to be due in part to the greater rhythmic skills of the present participants, though the cyclic nature of the rhythms may also have improved accuracy. Fraisse observed that when i2 and i3 were within the range of $300-600 \mathrm{~ms}$, I2 and I3 were assimilated to each other in production, while I1 was shortened. The assimilation was mainly due to lengthening of the shorter of the two longer intervals (I2, I3), especially when $\mathrm{i} 2<\mathrm{i} 3$ (in the left half of the graph). The present results for i $2<\mathrm{i} 3$ resemble Fraisse's, although here the I2-I3 assimilation region was a bit wider and included R3; shortening of I1 was observed even in R2. For i2 > i3 (right half of the graph), our data show assimilation of I 2 and I 3 in only one rhythm (R8), mainly in the musician group. Unlike Fraisse, we found that participants tended to dissimilate (i.e., contrast) I2 and I3 in R7, while I1 was produced very accurately in that rhythm. The I1 interval was also produced accurately in R6, where i $2=\mathrm{i} 3$. While rhythm experts produced that whole rhythm accurately, on average, musicians tended to make I3 shorter than I2 in synchronization.

Fraisse also found assimilation of I1 with I2 or I3 when i2 or i3 became shorter than $300 \mathrm{~ms}$, while at the same time the single remaining long interval was shortened. These tendencies were less pronounced in our data. We found shortening of the single long interval only when its duration was $750 \mathrm{~ms}$ (R1 and R11), and then only in synchronization. The two short intervals tended to be assimilated to each other in those two rhythms, and also in R10 by the musicians. The large interindividual variability in the duration of I2 in continuation of R9 and R10 in both participant groups should be noted. This interval, which separated the rhythmic groups created by the two short intervals (see R11 in Figure 2), seemed to be lengthened by some participants and shortened by others.

At the slow tempo (Figure 4), the pattern of deviations from the i2 and i3 target intervals was similar to that at the fast tempo, at least for the musicians. Correlations of their deviation patterns across tempi ranged from .71 to .78 , except for I2 in continuation, for which the correlation was zero. The musicians still showed assimilation of I2 and I3 in R3 and R4. Moreover, they showed again contrast of I2 and I3 in R7, although it was here accompanied by a lengthening of I1. Assimilation of the two short intervals near the extremes of the rhythm continuum was also evident. The rhythm experts showed few significant rhythm distortions, the only consistent one being the dissimilation of I2 and I3 and the simultaneous lengthening of I1 in R7.

The ANOVA on the Procrustes distances indicated that, on the whole, the rhythm experts produced the rhythms more accurately than did the musicians, $F(1,12)=5.43, p=.04$, and that rhythm production was relatively more accurate at the slow than at the fast tempo, $F(1,12)=6.93, p=.02$. The Tempo $\times$ Task interaction also reached significance, $F(1,12)=4.96$, $p<.05$. At the slow tempo, continuation was slightly more accurate than synchronization for both participant groups. At the fast tempo, the opposite held for musicians, while the rhythm experts showed no difference between tasks. However, the main effect of task and the triple interaction were not significant.

\section{INTERVAL VARIABILITY: SD-B}

In the ANOVAs on variability measures, rhythm was expected to have a strong linear effect on intervals I2 and I3, because variability is known to increase with interval duration (e.g., Peters, 1989), even in rhythmic contexts (e.g., Doumas \& Wing, 2007). Overlaid on this linear trend, however, higher-order nonlinear trends were expected, due to local increases in variability at the boundary between assimilation regions (cf. Figure 1B). Effects of expertise, tempo, and task were of interest as well. Rhythm experts were expected to be less variable than other musicians. If variability is roughly proportional to cycle duration, tempo should not have any main effect on variability (which was expressed proportional to cycle duration in the ANOVAs), though it might interact with other variables. Synchronization was expected to be more variable than continuation, due to necessary phase correction in synchronization. 
A

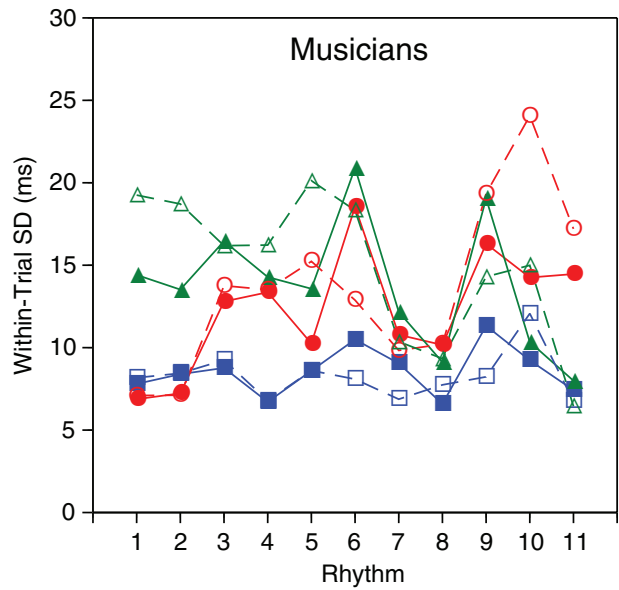

B

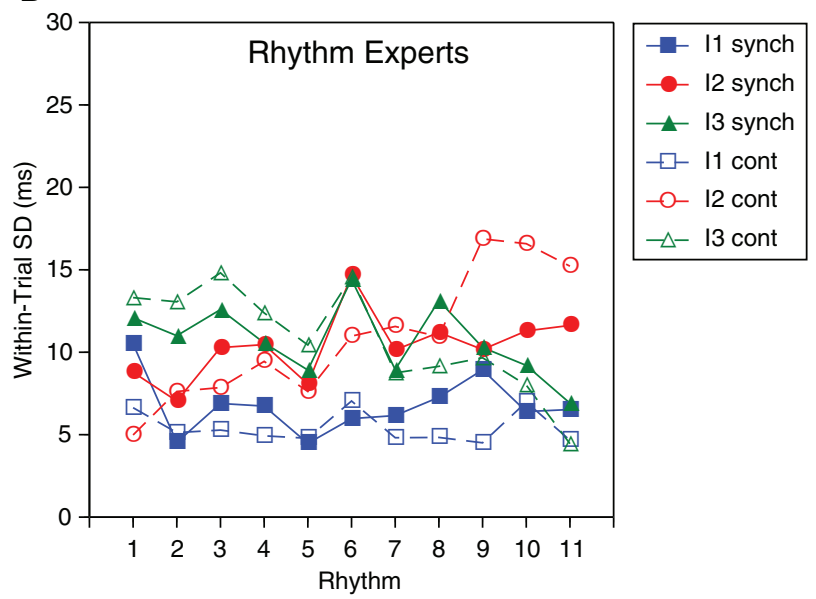

FIGURE. 5 Mean between-trial standard deviations of interval durations produced by musicians (panel A) and rhythm experts (panel B) in synchronization and continuation at the fast tempo.

Figures 5 and 6 show the $S D$-b values of the three intervals at the fast and slow tempo, respectively. As noted earlier, the $S D$-b reflects the within-participant variability of the mean interval durations (the rhythm "interpretation") across the five trials. Not surprisingly, this variability was generally lowest for I1, the consistently short interval, unless I2 or I3 was also very short. Despite the constancy of i1, the $S D$-b of I1 varied significantly with rhythm, $F(10,120)=3.29, p=.02$. There was a significant linear trend, $F(1,12)=5.88$, $p=.03$, because variability tended to increase with I3 duration (the preceding interval). However, five nonlinear contrasts were also significant, with the $5^{\text {th }}$ order contrast being the largest, $F(1,12)=14.87, p=.002$, suggesting a more intricate pattern. The effect of rhythm also interacted with task, $F(10,120)=3.46$,

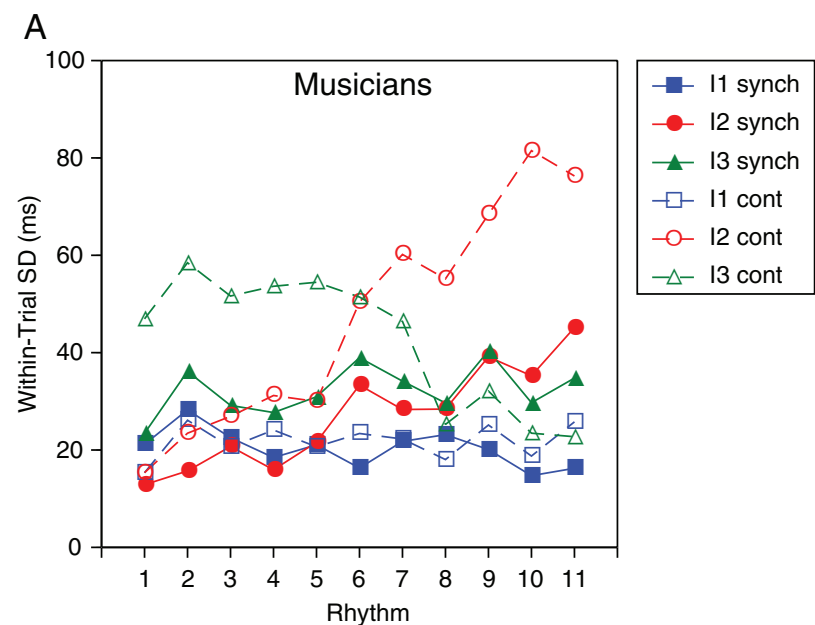

B

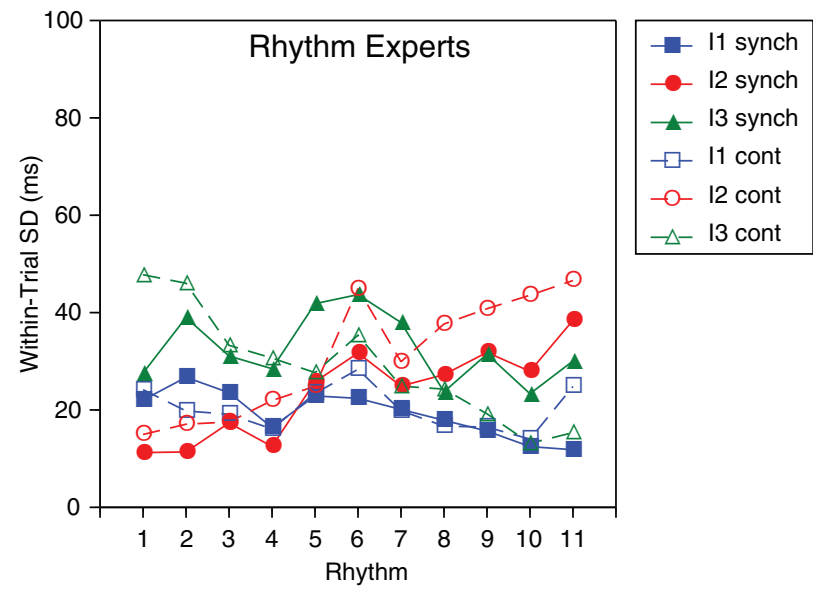

FIGURE. 6 Mean between-trial standard deviations of interval durations produced by musicians (panel A) and rhythm experts (panel $B$ ) in synchronization and continuation at the slow tempo.

$p=.008$. This interaction was located in the linear and quadratic contrasts. As can be seen in the figures, the increase in I1 variability with I3 duration seemed to occur only in synchronization, not in continuation. Furthermore, there was a pronounced main effect of task, $F(1,12)=55.89, p<.001$, which interacted with tempo, $F(1,12)=34.00, p<.001$. As expected, variability was larger in synchronization than in continuation, but this difference was much larger at the slow tempo, $F(1,12)$ $=56.09, p<.001$, than at the fast tempo, $F(1,12)=$ $12.86, p=.004$. The main effect of tempo and all effects involving expertise were nonsignificant.

The ANOVA on the $S D$-b of I2 revealed a significant effect of rhythm, $F(10,120)=18.90, p<.001$. As expected, there was a strong linear trend, $F(1,12)=$ $79.21, p<.001$, because $S D$-b increased with I2 
duration. However, there were four significant nonlinear contrasts as well, the largest being of $8^{\text {th }}$ order, $F(1,12)=28.20, p<.001$. The quadratic contrast was significant, $F(1,12)=16.12, p=.002$, because in addition to the linear trend, variability was higher inside the rhythm continuum than at its edges. (The detailed pattern will be discussed soon.) Rhythm also interacted with tempo, $F(12,120)=4.23, p=.003$. The interaction was located mainly in nonlinear contrasts ( $4^{\text {th }}$ and $5^{\text {th }}$ order). The main effect of rhythm was significant at each tempo, but at the fast tempo it included a number of significant nonlinear contrasts, whereas at the slow tempo these contrasts were less pronounced, indicating a less differentiated profile across rhythms. Rhythm furthermore interacted with task, $F(10,120)=5.56, p=.001$, and this interaction was entirely linear, $F(1,12)=29.15, p<$ .001 . The variability of I2 increased more steeply with I2 duration in continuation than in synchronization and increasingly exceeded the latter, which also resulted in a significant main effect of Task, $F(1,12)=12.03, p=$ .005 . Finally, there was one significant effect involving expertise, namely the Expertise $\times$ Tempo $\times$ Task interaction, $F(1,12)=12.27, p=.004$ : While musicians showed a larger difference between synchronization and continuation at the slow than at the fast tempo, the rhythm experts showed the opposite. However, the Expertise $\times$ Task interaction did not reach significance at either tempo, and continuation was (unexpectedly) always more variable than synchronization. The main effects of expertise and tempo were not significant.

I3 was expected to exhibit a $S D$-b pattern mirroring that of I2, which it largely did. The main effect of rhythm was significant, $F(10,120)=17.00, p<.001$, and included significant linear, quadratic, and higherorder nonlinear contrasts. The Rhythm $\times$ Tempo interaction was not significant here, but the Rhythm $\times$ Task interaction was significant, $F(10,120)=8.15, p<.001$, and was again purely linear, $F(1,12)=103.67, p<.001$, due to a stronger increase of $S D$-b with I3 duration in continuation than in synchronization. This difference was more pronounced at the fast than at the slow tempo, accounting for a significant Rhythm $\times$ Tempo $\times$ Task interaction, $F(10,120)=2.56, p=.04$. Nevertheless, the Rhythm $\times$ Task interaction was significant at both tempi. The main effect of task was also significant, $F(1,12)=5.14, p=.04$, due to higher variability in continuation than in synchronization. The main effect of tempo reached significance here, $F(1,12)=7.80$, $p=.01$, reflecting relatively higher variability at the slow than at the fast tempo. Finally, as for I2, the Expertise $\times$ Tempo $\times$ Task interaction was significant, $F(1,12)=10.11, p=.008$, reflecting a similar pattern of differences as for I2. The main effect of expertise was not significant.

Comparing now the variability profiles to the single composite profile reported by Fraisse (Figure 1B), we note one striking difference: Here there was a pronounced peak in both I2 and I3 variability for the target rhythm in which i2 = i3 (R6), in both synchronization and continuation and for both participants groups, whereas Fraisse observed low variability for that rhythm. ${ }^{6}$ Thus it appears that I2 and I3 were often produced with unequal durations when their target durations were the same, though not in a consistent manner across trials.

The musicians tended to show two additional variability peaks for both I2 and I3, located at R3 and R9. Taking into account that the region of assimilation of I2 and I3 (excepting R7, which showed contrast) was a bit broader here than in Fraisse's results, these variability peaks correspond to the boundary between that region and the region where the two shorter intervals tended to be assimilated to each other, consistent with Fraisse's findings. They imply that individual participants varied from trial to trial in how they treated the boundary rhythms, sometimes assimilating I2 with I3 and at other times, I1 with the shorter of I2 and I3. Our rhythm experts did not exhibit any clear boundary peaks, but since the Expertise $\times$ Rhythm interaction was not significant in the ANOVAs, not much can be made of that group difference.

\section{INTERVAL VARIABILITY: SD-W}

The $S D$-w values at the two tempi, shown in Figures 7 and 8 , were much smaller than the corresponding $S D$-b values in Figures 5 and 6 (note the different $y$-axis scale), which suggests that whatever rhythm "interpretation" (i.e., particular distortion pattern) participants adopted in a given trial, they generally stuck to it throughout the trial. Naturally, I1 variability was smaller overall than I2 and I3 variability, due to its consistently short duration. For I1, there were no significant effects involving rhythm. Only one effect was significant for I1, the main effect of task, $F(1,12)=27.45, p<.001$ : Variability was larger in synchronization than in continuation, as predicted.

I2 variability exhibited a significant main effect of rhythm, $F(10,120)=17.31, p<.001$. In contrast to the $S D$-b results, however, that effect was predominantly linear, $F(1,12)=77.51, p<.001$. Only two nonlinear

\footnotetext{
${ }^{6}$ We examine here within-participant variability, whereas Fraisse's figure shows between-participant variability. However, Fraisse mentioned (p. 54) that he found a similar pattern when examining within-participant variability. Relatively high between-participant variability of R6, compared to R5 and R7, can also be seen in the error bars of our data in Figures 3 and 4.
} 
A

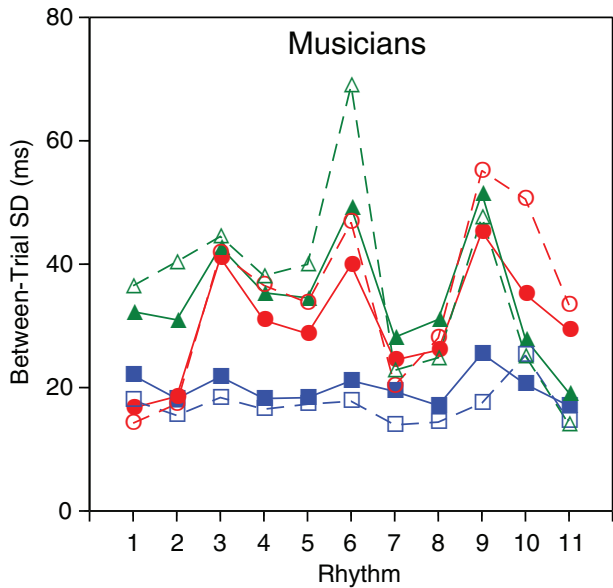

B

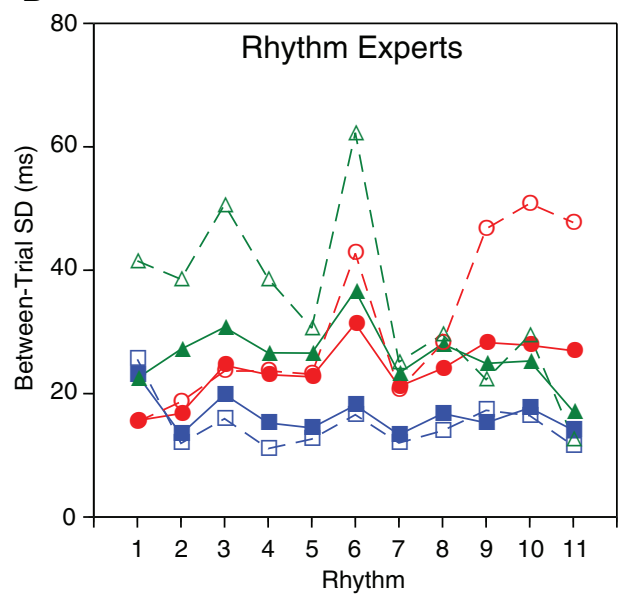

FIGURE. 7 Mean within-trial standard deviations of interval durations produced by musicians (panel A) and rhythm experts (panel B) in synchronization and continuation at the fast tempo.

contrasts reached significance, the quadratic, $F(1,12)=$ $7.26, p=.02$, and the $8^{\text {th }}$ order, $F(1,12)=5.98, p=.03$. $S D$-w increased especially clearly with I2 duration at the slow tempo, but the Rhythm $\times$ Tempo interaction did not reach significance, nor did the Rhythm $\times$ Task interaction. There was a main effect of task, $F(1,12)=8.84$, $p=.01$, reflecting (again unexpectedly) higher variability in continuation than in synchronization, and a Tempo $\times$ Task interaction, $F(1,12)=5.99, p=.03$, because the task difference was more pronounced at the slow than at the fast tempo. Finally, this interaction further interacted with expertise, $F(1,12)=10.77$, $p=.007$, because the task difference was largest for musicians at the slow tempo. Indeed, the Expertise $x$ Task interaction was significant only at the slow tempo, $F(1,12)=5.84, p=.03$. The main effects of expertise and tempo were not significant.
A

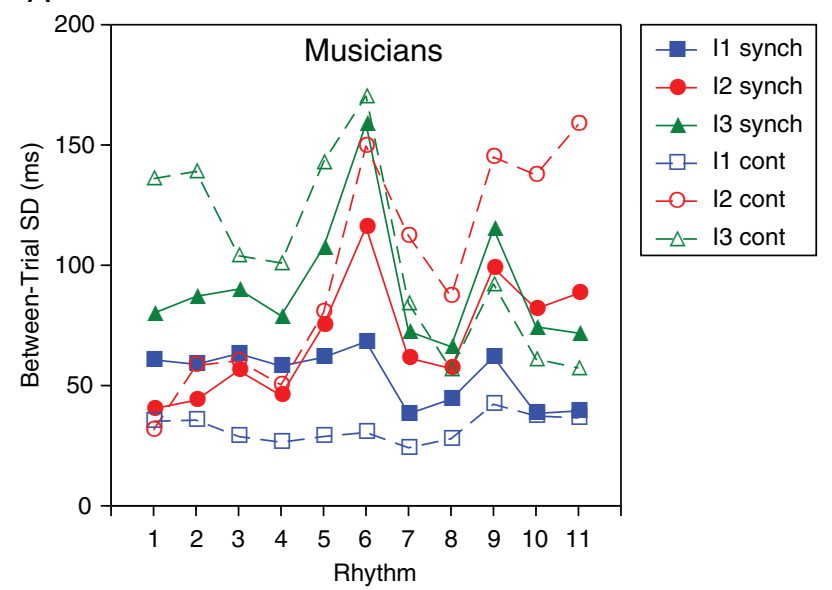

B

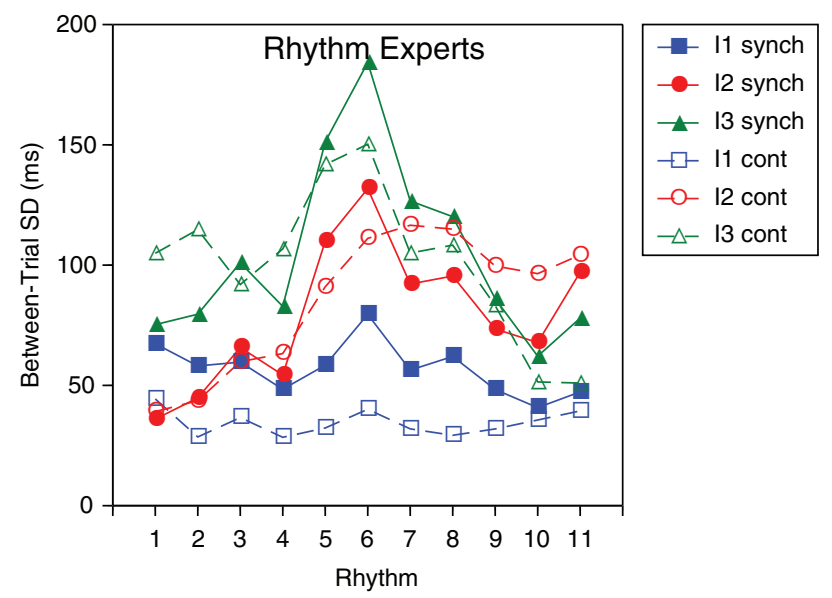

FIGURE. 8 Mean within-trial standard deviations of interval durations produced by musicians (panel A) and rhythm experts (panel B) in synchronization and continuation at the slow tempo.

For I3, we found again a main effect of rhythm, $F(12,120)=7.92, p<.001$, with a strong linear increase of $S D$-w with I3 duration, $F(1,12)=39.37, p<.001$, but with clearly significant nonlinear contrasts as well (quadratic, $6^{\text {th }}$ and $8^{\text {th }}$ order). Rhythm interacted with task, $F(10,120)=3.58, p=.007$, again in a predominantly linear fashion, $F(1,12)=25.68, p<.001$, because the increase with I3 duration was more pronounced in continuation than in synchronization. Indeed, musicians showed no increase at all at the slow tempo (Figure $8 \mathrm{~A}$ ), but the triple interaction with expertise was far from significance. The main effect of task also fell short of significance, but there was a Tempo $\times$ Task interaction, $F(1,12)=6.51, p=.03$, because a significant difference between tasks was observed only at the slow tempo, $F(1,12)=5.53, p=.04$. No other effects were significant. 
The pattern of $S D$-w across rhythms was similar to that of $S D$-b, indicating greater cycle-to-cycle instability of some rhythms than others. Again there was a variability peak in the middle of the continuum (R6), and only the musicians tended to show side peaks (R3, R9) as well, though the Expertise $\times$ Rhythm interaction was not significant.

\section{Discussion}

The present results partially replicate Fraisse's finding of a tendency for the two longer intervals or the two shorter intervals to be assimilated in reproduction of threeinterval rhythms, presumably depending on whether the intermediate interval is perceived as relatively long or short. Whereas Fraisse had nonmusicians reproduce a single rhythmic group, we asked highly trained musicians including percussionists to reproduce rhythms cyclically in synchrony with exact auditory rhythm templates, and then to continue to produce them without such templates. The persistence of assimilation tendencies under these stringent conditions, especially at the faster tempo (the tempo also used by Fraisse), demonstrates their wellnigh obligatory nature. Even though, due to the rhythmic expertise of the participants and perhaps also due to the cyclic repetition of the rhythms, the tendencies were smaller here than in Fraisse's study and hardly ever resulted in complete assimilation, they were highly reliable statistically, which means they were shown by most participants. Many participants commented after the experiment that they found the task difficult, especially at the slow tempo, though they were probably more aware of their variability than of their constant errors.

We also partially replicated Fraisse's finding of a tendency to shorten the short interval when the other two intervals are comparatively long, though we found this only in synchronization at the fast tempo (the tempo also used by Fraisse). Furthermore, like Fraisse, we found inconsistency in production of rhythms whose intermediate interval was neither clearly short nor clearly long. This was reflected in the "boundary peaks" in the variability patterns of the musician group. It appears that these participants assimilated the intermediate interval to the short interval in some trials, and to the long interval in others. All these results seem to support Fraisse's contention that three-interval rhythms gravitate towards structures in which there are only two distinct interval durations.

However, this conclusion is challenged by two results that deviate from Fraisse's findings. First, we found that the most variably reproduced target rhythm was the one in which the two long intervals were identical. Fraisse found low variability for that rhythm, as should be the case if participants aim for two distinct interval categories. Our different result is likely to be due to the fact that our rhythms were produced cyclically: Perhaps participants needed one interval to be the longest, so as to serve as a separator of three-tone rhythmic groups, and they sometimes chose I2 and at other times I3. Second, there was one rhythm (R7) in which the two long intervals were contrasted rather than assimilated in production. The fact that there was only one such rhythm means that the order of intervals of the same duration played a role, with i2 > i3 (R7), but not i2 < i3 (R5), eliciting contrast. The cyclic format may also be responsible for this difference, as discussed below.

Fraisse's results, showing strong assimilation of I2 and I 3 and a categorical tendency (similar reproduction of different rhythms) in the center of the rhythm continuum (Figure 1A), suggest a single "attractor" in rhythm space, namely one identical with or in the vicinity of R6. The interval ratios of R6 are not simple (2:5:5), but they satisfy the requirement of two distinct interval categories. However, such ratios may not fare well in cyclic reproduction, where regularizing metrical tendencies come to the fore.

Instead of a single attractor in the center of the rhythm continuum, our results instead suggest two attractors, to the left and right of center, respectively. This is indicated not only by the high variability of R6 but also by categorical tendencies in the form of local plateaus at R4-R5 and R7-R8, respectively. The rhythms in these pairs tended to be reproduced similarly, as if they were assimilated to an attractor located between them. Significantly, the rhythms halfway between each of these pairs (which we did not actually present, though Fraisse did) have simple interval ratios of $1: 2: 3(180 / 360 / 540 \mathrm{~ms})$ and 1:3:2 (180/540/360 ms), respectively. Thus they represent the simplest, most metrical rhythms with three different interval durations, given il $=180 \mathrm{~ms}<\mathrm{i} 2$, i3.

The evidence for a 1:3:2 attractor (between R7 and R8) is strongest at the fast tempo, especially for the rhythm experts. The dissimilation of I2 and I3 in R7 in combination with the assimilation of I2 and I3 in R8 led to nearly horizontal lines connecting their I2 and I3 durations, which intersect the dotted target line in Figure 3. Such points of intersection represent an attractor, defined as a rhythm that would have been reproduced perfectly (on average) if it had actually been presented. For the rhythm experts, the intersection points at the fast tempo are very close to the 1:3:2 rhythm, halfway between R7 and R8. For the musicians, they are closer to R7. At the slow tempo, the intersections are close to R8 for the musicians, while the rhythm experts show hardly 
any attractor effect. If these deviations of the intersections from the 1:3:2 attractor were statistically reliable (we did not assess this), they would suggest an attractor in the vicinity of 1:3:2 whose interval ratios are not simple. In studies of musicians' production and perception of two-interval rhythms, Repp et al. $(2011,2012)$ recently found that the attractor deviated significantly from 1:2. Similar deviations from simple-ratio attractors for three-interval rhythms thus are conceivable.

The data for R4-R5 also suggest an attractor with complex interval ratios, in the vicinity of 1:2:3 but not exactly 1:2:3, because both R4 and R5 exhibited assimilation of I2 and I3. The best estimate of this attractor would presumably be the midpoint of the lines connecting the mean I2 and I3 (and I1) durations of R4 and R5. It should be noted that I2-I3 assimilation was generally stronger when i2 < i3 (R3-R5) than when i2 $>$ i3 (R8 only). This indicates a stronger attractor in the first case, albeit one with complex interval ratios. This asymmetry between the left and right sides of the rhythm continuum can be explained by considering the underlying metrical structure that these rhythms may give rise to. The tone preceding il (C4), though nominally the first tone in the rhythm cycle, is likely to be interpreted as an upbeat to the following tone (D4), which then probably functions as the downbeat initiating metrical cycles ("measures") unless i3 is also short, in which case E4 may become the downbeat. Thus, the real attractors for rhythms in the vicinity of 1:3:2 (R7, R8) may have been $3: 2: 1$ or $2: 1: 3$, metrical patterns that comfortably fit a duple meter with triple subdivision $(3+3$, usually notated as 6/8). Similarly, rhythms in the vicinity of 1:2:3 (R4, R5) may have activated a 2:3:1 or 3:1:2 attractor. These metrical patterns, however, are syncopated in either a duple or a triple meter (cf. Repp et al., 2002). Thus they may be weaker attractors. This may explain why these attractors seem to deviate more from simple interval ratios. Perhaps, when a target rhythm is fit less easily into a metrical scheme, the nonmetrical "Fraisse attractor," which merely wants to distinguish two interval categories regardless of ratio, comes into play and competes with the metrical attractor. Apparently distorted metrical attractors thus may reflect the simultaneous operation of metrical and nonmetrical attractors.

The assimilation of the two short intervals near the extremes of our (and Fraisse's) rhythm continuum at the fast tempo could be explained in terms of metrical attractors located near 180/180/720 and 180/720/180 $\mathrm{ms}$, which represent 1:1:4 and 1:4:1 interval ratios. Because three tones separated by two short intervals form a tight rhythmic group in which the first and last tones are more salient than the middle tone (Povel \&
Essens, 1985), both of these attractor rhythms are effectively 1:1:4 or 4:1:1, either of which fits a triple meter with duple subdivision $(2+2+2$, commonly notated as 3/4). Indeed, 1:1:4 was a salient category in the context of an induced triple meter in Desain and Honing's (2003) rhythm categorization study. The 1:1:4 attractor could also be considered a two-interval rhythm attractor with a $(1+1): 4=1: 2$ interval ratio (or thereabouts), which is the dominant attractor in production of two-interval rhythms (Povel, 1981; Repp et al., 2011).

It thus seems that the pattern of rhythm distortions we observed can be interpreted rather well in terms of metrical rhythm categories representing nominally simple interval ratios. This resolves the apparent conflict with the Desain and Honing (2003) categorization data noted earlier, where the dominant categories (1:1:2, 1:2:1, 2:1:1) pertained only to duple meter with duple subdivision $(2+2$, notated as $2 / 4)$. Of course, we do not know for sure that our participants interpreted the rhythms as being in $6 / 8$ or $3 / 4$ meter. It would be interesting to re-examine production of these rhythms in the context of unambiguous duple and triple metrical frameworks. In the present experiment, participants presumably chose a metrical interpretation (if any) that they found congenial when aiming for accuracy of reproduction. This may not have been a conscious choice; rather, a preferred metrical framework may emerge automatically from repeated exposure to a rhythm, and it may differ for different rhythms.

Our experiment yielded a few secondary results of interest, some of them unexpected. As expected, rhythm distortions were relatively smaller at the slow than at the fast tempo, but the patterns were similar. In his analysis of rhythmic structures, Fraisse (1956) distinguished between categories of short and long intervals, with the boundary being around 300-400 ms. If the boundary were drawn at a shorter duration (200-300 ms), Fraisse's distinction would correspond to a distinct break in the function relating interval duration and the discrimination threshold (see, e.g., Friberg \& Sundberg, 1995). When our rhythms were presented at the fast tempo, i1 $(180 \mathrm{~ms})$ clearly was short in Fraisse's sense, whereas i2 and i3 were either both long, or one was long and the other short, or their long-short status was ambiguous. In our results, assimilation of I2 and I3 ceased only when the shorter of these intervals approached $200 \mathrm{~ms}$ (cf. Figure 3), which suggests a rather low short-long category boundary. At the slow tempo, however, all target intervals were long in Fraisse's sense (i1 $=450 \mathrm{~ms}$; i2, i3 $\geq 375 \mathrm{~ms}$ ), which may have reduced the tendency to form two distinct interval categories. Nevertheless, the pattern of 
distortions was similar to that at the fast tempo, though weaker. Thus the boundary between the short and long categories may be flexible and shift with tempo.

In any case, in view of the high variability of reproduction of the rhythm (R6) that most clearly instantiated just two interval categories, Fraisse's hypothesis of two interval categories now seems too simplistic as an explanation of the assimilation tendencies in cyclic rhythms. Our pattern of results mainly seems to reflect attraction to ideal rhythms in which all three intervals are different and have (close to) simple ratios, as is common in metrical contexts. The finding of smaller rhythm distortions at a slow tempo than at a fast tempo could be due to the longer separations between events, which reduces the temporal coherence of the rhythms and thus their rhythmicity and metricality, thereby weakening the influence of metrical attractors. Metrical attractors may also be weakened, however, by a very fast tempo. If that were not the case, it would be difficult to explain the deviations from simple interval ratios in the study of Repp et al. (2002), where pianists played notated three-interval rhythms that represented the hypothetical attractors (i.e., having 1:2:3 interval ratios in various permutations). Cycle durations in that study ranged from 600 to 1500 $\mathrm{ms}$, as compared to $1080 \mathrm{~ms}$ at the fast tempo here, and deviations from the target rhythms were observed mainly at the two fastest tempi. As cycle durations get shorter, their subdivision into beats is impeded (see London, 2004), and eventually metrical structure within cycles is obliterated and the cycles themselves become the beats. Thus, the assimilation of the two longer intervals that Repp et al. observed at fast tempi can be attributed again to a nonmetrical Fraisse attractor. Metrical attractors are likely to be strongest at moderate tempi that allow easy subdivision of cycles into regular beats.

Interval variability generally increases with interval duration in both isochronous and non-isochronous rhythmic contexts (Doumas \& Wing, 2007; Semjen \& Ivry, 2001), and the present data confirmed this linear trend, although it was overlaid and severely disturbed by variability due to rhythmic instability, particularly at the fast tempo. Thus, the present results show that the particular configuration of interval durations in a cyclical rhythm can be an important determinant of interval variability in addition to interval duration itself. Even the variability of I1 varied with the duration of the preceding interval (I3). In the context of synchronization this could be regarded as a reflection of phase correction. Indeed, as predicted, variability of I1 was greater in synchronization than in continuation, most strikingly so at the slow tempo. The variability of $\mathrm{I} 2$ and I3, however, was greater in continuation than in synchronization, which suggests that the variability of these (usually longer) intervals reflected memory instability more than presence versus absence of phase correction. Another somewhat surprising result was that $S D$-b was much larger than $S D$-w. This means that participants varied more in how they reproduced a given target pattern from trial to trial than they varied from cycle to cycle within a trial, implying that in each trial they arrived at a somewhat different "interpretation" of the rhythm and maintained that interpretation throughout the trial. If their interpretation had not varied, they should have converged on the same mean interval durations in each trial, resulting in $S D$-b $<S D$-w.

Although we expected rhythm experts (mostly percussionists) to be more accurate and less variable than other musicians, this prediction was borne out only partially. The experts generally had smaller constant errors, but their variability was lower only within trials at the fast tempo. At the slow tempo, with the rhythms being less rhythmic as it were, rhythm expertise seemed to confer less of an advantage in terms of variability. There was clearly overlap between the two participant groups: Not all rhythm experts were more accurate and less variable than all other musicians. At the fast tempo, it did not seem that any rhythm expert could avoid deviations from the target rhythms. At the slow tempo, some of them may have succeeded in reproducing the target rhythms accurately on average, but with considerable variability.

In conclusion, the present study extends Fraisse's classical findings of interval assimilation in threeinterval rhythms by showing that such assimilation also occurs in cyclic rhythm production by highly trained musicians, even in synchronization with an exact rhythm template. Thus these temporal distortions seem to be quite compulsory. Unlike Fraisse's data, which suggested a simple tendency to reduce three interval categories to two, our results suggest metrical attractors having three different interval durations, with close to simple ratios. We attribute this difference in results to the cyclic nature of our rhythms. However, the nonmetrical attractor identified by Fraisse may still have some effect even in cyclic rhythms, and this may account for apparent distortions in the metrical attractors themselves.

\section{Author Note}

This research was supported by National Science Foundation grant BCS-0924206 (BHR) and by the Max Planck Society (PEK).

Correspondence concerning this article should be addressed to Bruno H. Repp, e-mail: repp@haskins.yale .edu 


\section{References}

$\rightarrow$ Desain, P., \& Honing, H. (2003). The formation of rhythmic categories and metric priming. Perception, 32, 341-365.

$\rightarrow$ Doumas, M., \& Wing, A. M. (2007). Timing and trajectory in rhythm production. Journal of Experimental Psychology: Human Perception and Performance, 33, 442-455.

Dryden, I. L., \& Mardia, K. V. (1998). Statistical shape analysis. Chichester, UK: Wiley.

Fraisse, P. (1956). Les structures rythmiques [Rhythmic structures]. Louvain, Belgium: Publications Universitaires de Louvain.

$\rightarrow$ Friberg, A., \& Sundberg, J. (1995). Time discrimination in a monotonic, isochronous sequence. Journal of the Acoustical Society of America, 98, 2524-2531.

$\rightarrow$ Gabrielsson, A., Bengtsson, I., \& Gabrielsson, B. (1983). Performance of musical rhythm in $3 / 4$ and $6 / 8$ meter. Scandinavian Journal of Psychology, 24, 193-213.

London, J. (2004). Hearing in time: Psychological aspects of musical meter. New York: Oxford University Press.

$\rightarrow$ Peters, M. (1989). The relationship between variability of intertap intervals and interval duration. Psychological Research, 51, 38-42.

$\rightarrow$ Povel, D.-J. (1981). Internal representation of simple temporal patterns. Journal of Experimental Psychology: Human Perception and Performance, 7, 3-18.

$\rightarrow$ Povel, D.-J., \& Essens, P. (1985). Perception of temporal patterns. Music Perception, 2, 411-440.

$\rightarrow$ Repp, B. H., London, J., \& Keller, P. E. (2005). Production and synchronization of uneven rhythms at fast tempi. Music Perception, 23, 61-78. $\rightarrow$ Repp, B. H., London, J., \& Keller, P. E. (2008). Phase correction in sensorimotor synchronization with nonisochronous sequences. Music Perception, 26, 171-175.

$\rightarrow$ Repp, B. H., London, J., \& Keller, P. E. (2011). Perceptionproduction relationships and phase correction in synchronization with two-interval rhythms. Psychological Research, 75, 227-242.

$\rightarrow$ Repp, B. H., London, J., \& Keller, P. E. (2012). Distortions in reproduction of two-interval rhythms: When the "attractor ratio" is not exactly 1:2. Music Perception, 30, 205-223.

$\rightarrow$ Repp, B. H., Windsor, L., \& Desain, P. (2002). Effects of tempo on the timing of simple musical rhythms. Music Perception, 19, 565-593.

$\rightarrow$ Sadakata, M., Desain, P., \& Honing, H. (2006). The Bayesian way to relate rhythm perception and production. Music Perception, 23, 269-288.

$\rightarrow$ Semjen, A., \& Ivry, R. B. (2001). The coupled-oscillator model of between-hand coordination in alternate-hand tapping: A reappraisal. Journal of Experimental Psychology: Human Perception and Performance, 27, 251-265.

$\rightarrow$ Snyder, J. S., Hannon, E. E., Large, E. W., \& Christiansen, M. H. (2006). Synchronization and continuation tapping to complex meter. Music Perception, 24, 135-146.

$\rightarrow$ Summers, J. J., Bell, R., \& Burns, B. D. (1989). Perceptual and motor factors in the imitation of simple temporal patterns. Psychological Research, 50, 23-27.

$\rightarrow$ Summers, J. J., Hawkins, S. R., \& Mayers, H. (1986). Imitation and production of interval ratios. Perception and Psychophysics, 39, 437-444. 
This content downloaded from 194.95.183.252 on Thu, 22 Jan 2015 03:49:47 AM

All use subject to JSTOR Terms and Conditions 